\title{
Search for Turbulent Gas through Interstellar Scintillation
}

\author{
M. Moniez ${ }^{1}$, R. Ansari ${ }^{1}$, F. Habibi ${ }^{1}$, and S. Rahvar ${ }^{2,3}$ \\ ${ }^{1}$ LAL, IN2P3-CNRS, Université de Paris-Sud, 91898 Orsay Cedex, France \\ email: moniez@lal.in2p3.fr \\ ${ }^{2}$ Department of Physics, Sharif University of Technology, Tehran, Iran \\ ${ }^{3}$ Perimeter Institute for Theoretical Physics, Waterloo, Ontario N2L 2Y5, Canada
}

\begin{abstract}
Stars twinkle because their light propagates through the atmosphere. The same phenomenon is expected when the light of remote stars crosses a Galactic-disk or halorefractive medium such as a molecular cloud. We present the promising results of a test performed with the ESO-NTT, and consider its potential.
\end{abstract}

Keywords. Galaxy:structure, dark matter, ISM

\section{What is Interstellar Scintillation?}

Refraction through an inhomogeneous transparent cloud ("screen") distorts the wavefront of incident electromagnetic waves (Fig. 1). For a point-like source the intensity in the observer's plane is affected by interference which, in the case of stochastic inhomogeneities, causes it to take on a speckle appearance that is characterized by at least two distance scales:

- The diffusion radius $R_{d i f f}(\lambda)$ of the screen, defined as the transverse separation for which the root mean square of the phase difference at wavelength $\lambda$ is 1 radian, and

- The refraction radius

$$
R_{\text {ref }}(\lambda)=\frac{\lambda z_{0}}{R_{\text {diff }}} \sim 30860 k m\left[\frac{\lambda}{1 \mu m}\right]\left[\frac{z_{0}}{1 k p c}\right]\left[\frac{R_{d i f f}(\lambda)}{1000 k m}\right]^{-1},
$$

where $z_{0}$ is the distance to the screen. This is the size, in the observer's plane, of the diffraction spot from a patch of $R_{d i f f}(\lambda)$ in the screen's plane.

After crossing a fractal cloud described by the Kolmogorov turbulence law (Fig. 1, left), the light from a monochromatic point source produces an illumination pattern on Earth consisting of speckles of size $R_{d i f f}(\lambda)$ within larger structures of size $R_{r e f}(\lambda)$ (Fig. 1 , right). The illumination pattern from a stellar source of radius $r_{s}$ is the convolution of the point-like intensity pattern with the projected intensity profile of the source (Fig. 2, upper right).

A cloud moving with a transverse velocity $V_{T}$ relative to the line of sight will induce stochastic intensity fluctuations with amplitude predicted by Fig. 3 at the characteristic time-scale

$$
t_{\text {ref }}(\lambda)=\frac{R_{\text {ref }}(\lambda)}{V_{T}} \sim 5.2 \text { minutes }\left[\frac{\lambda}{1 \mu m}\right]\left[\frac{z_{0}}{1 k p c}\right]\left[\frac{R_{d i f f}(\lambda)}{1000 \mathrm{~km}}\right]^{-1}\left[\frac{V_{T}}{100 \mathrm{~km} / \mathrm{s}}\right]^{-1} .
$$

Signature of the scintillation signal. The first two signatures point to a propagation effect, which is incompatible with any type of intrinsic source variability.

- Chromaticity: Since $R_{r e f}$ depends on $\lambda$, one expects a variation in the characteristic time-scale $t_{\text {ref }}(\lambda)$ between the red side of the optical spectrum and the blue side. 

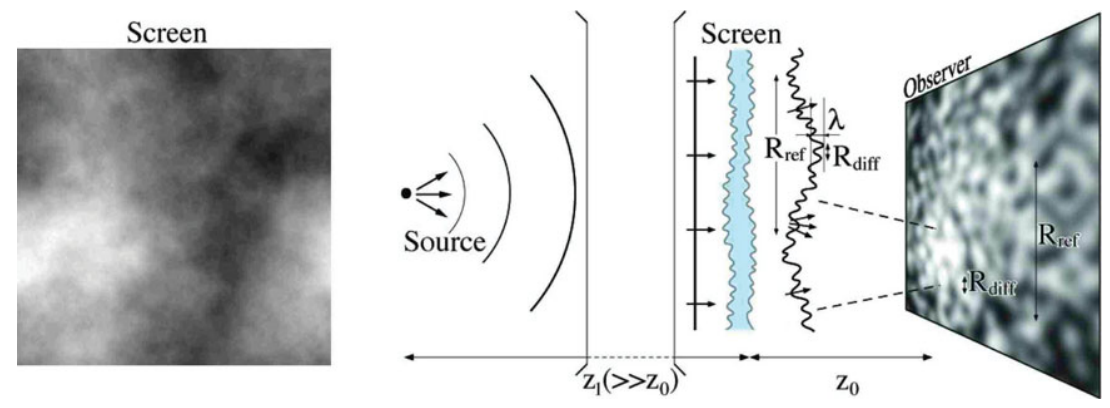

Figure 1. Left: A 2D stochastic phase screen (grey scale), from a simulation of gas affected by Kolmogorov-type turbulence. Right: The illumination pattern from a point source (left) after crossing such a phase screen. The distorted wavefront produces structures at scales $\sim R_{\text {diff }}(\lambda)$ and $R_{r e f}(\lambda)$ in the observer's plane.
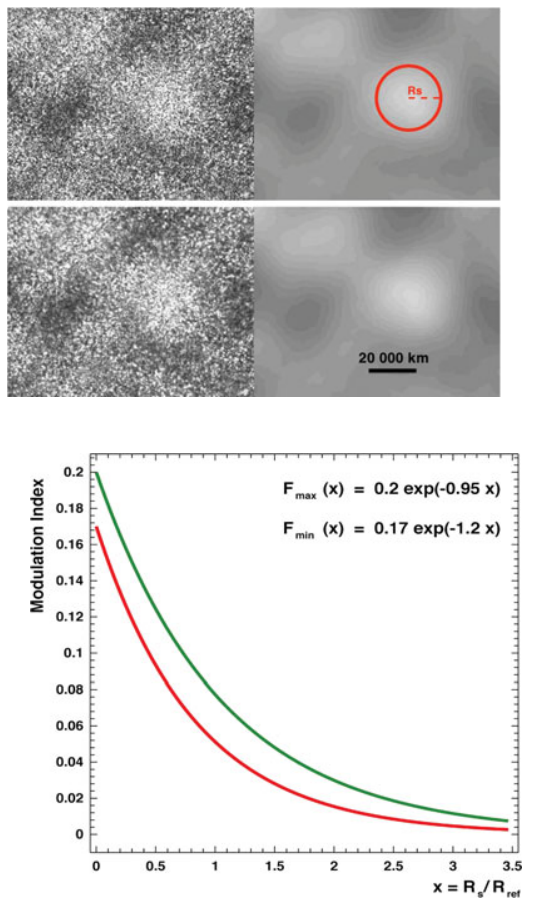

Figure 2. Simulated illumination map at $\lambda=2.16 \mu \mathrm{m}$ on Earth from a point source (upper left) and from a K0 V star $\left(r_{s}=0.85 R_{\odot}, M_{V}=5.9\right)$ at $z_{1}=8 \mathrm{kpc}$ (right). The refracting cloud is assumed to be at $z_{0}=160 \mathrm{pc}$ with a turbulence parameter $R_{\text {diff }}(2.16 \mu \mathrm{m})=150 \mathrm{~km}$. The circle shows the projection of the stellar disk (with radius $R_{S}=r_{s} \times z_{0} / z_{1}$ ). The bottom maps are illuminations in the $K_{s}$ wide band $\left(\lambda_{\text {central }}=2.162 \mu \mathrm{m}\right.$, $\Delta \lambda=0.275 \mu m)$.

Figure 3. The intensity modulation index $m_{\text {scint }}=\sigma_{I} / \bar{I}$ decreases when the ratio of the projected stellar disk $R_{S}$ to the refraction scale $R_{\text {ref }}(\lambda)$ increases. The modulation index is contained between the curves represented by functions $F_{\min }(x)$ and $F_{\max }(x)$.

- Spatial de-correlation: We expect a de-correlation between the light-curves observed at different telescope sites, increasing with their distance.

- Correlation between the stellar radius and the modulation index: Big stars scintillate less than small stars through the same gaseous structure.

- Location: The probability for scintillation is correlated with the foreground gas column density. Extended structures may therefore induce clusters of neighbouring scintillating stars.

Foreground effects, background to the signal. Atmospheric intensity scintillation is negligible through a large telescope (Dravins et al. 1997, 1998). Any other atmospheric effect should be easy to recognize as it affects all stars. Asteroseismology, granularity of the stellar surface, spots or eruptions produce variations of very different amplitudes and time-scales. A rare type of recurrent variable star exhibits emission variations on the minute scale, but such objects could be identified from their spectra. 

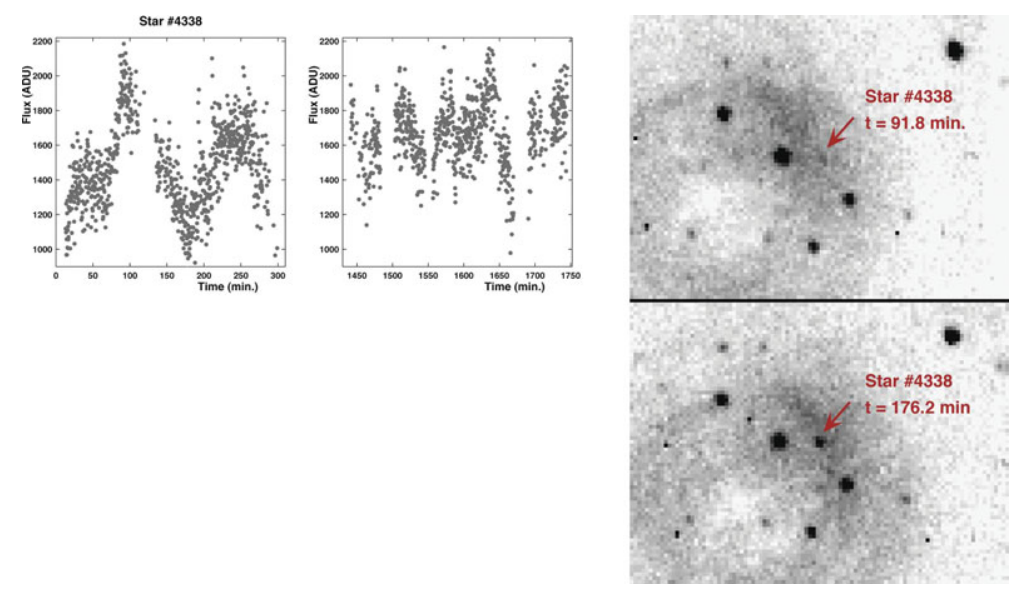

Figure 4. Above: Light-curves for the two nights of observation. Right: images of the candidate found toward B68 during a low-luminosity phase (upper) and a high-luminosity phase (lower); North is up, East is left.

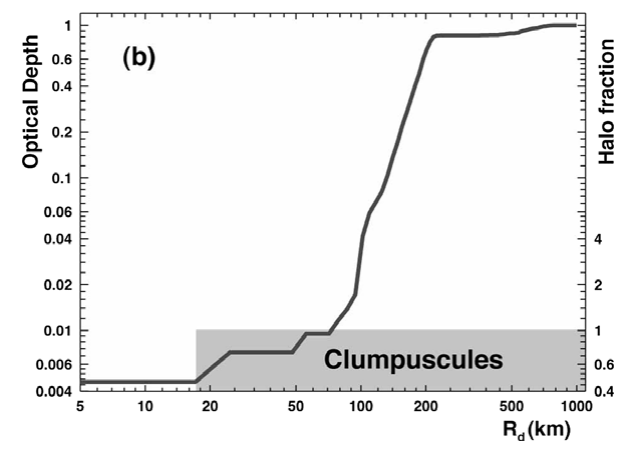

Figure 5. The $95 \% C L$ maximum optical depth of structures with $R_{d i f f}<R_{d}$ towards the SMC. The right scale gives the maximum contribution of structures with $R_{\text {diff }}(1.25 \mu \mathrm{m})<R_{d}$ to the Galactic halo (in fraction); the grey zone shows the possible region for the hidden gas clumpuscules expected from the model of Pfenniger \& Combes (1994).

\section{Preliminary Studies with the NTT, and Future Prospects}

During two nights in 2006 June, 4749 consecutive exposures of $T_{\text {exp }}=10 \mathrm{~s}$ were made with the infra-red SOFI detector in $K_{s}$ and $J$ through nebulae B68, cb131, Circinus and towards the SMC (Habibi et al. 2011). A candidate was found towards B68 (Fig. 4), but the poor photometric precision in $K_{s}$ and other limitations prevented definitive conclusions. Nevertheless, we can surmise from the rarity of stochastically fluctuating objects that there is no significant population of stars that can mimic scintillation effects, and future searches should not be overwhelmed by a background of fakes.

From the observed SMC light-curves we also established upper limits (not yet competitive) on invisible gaseous structures as a function of their diffusion radius (Fig. 5). Those constraints are limited by the statistics and by the photometric precision.

Prospects. LSST will be an ideal set-up to search for this signature of gas, thanks to the fast readout and to the wide and deep field. Scintillation signal would provide a new tool to measure the inhomogeneities and dynamics of nebulæ, and to probe the molecular hydrogen contribution to the baryonic hidden matter of the Milky Way.

\section{References}

Dravins, D., Lindegren, L., Mezey, E., \& Young, A. T. 1997, PASP, 109, 173; 109, 725

Dravins, D., Lindegren, L., Mezey, E., \& Young, A. T. 1998, PASP, 110, 610

Habibi F., Moniez M., Ansari R., \& Rahvar S. 2011, A\&A, 525, 108

Pfenniger, D. \& Combes, F. (1994) A\&A, 285, 94 